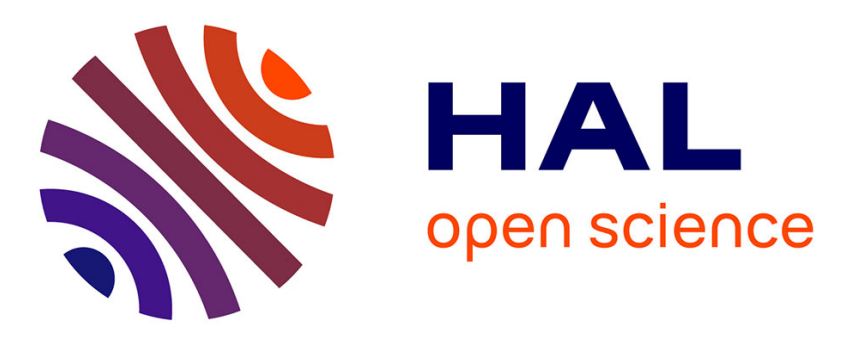

\title{
On the stabilization of high order systems with two unstable poles plus time delay
}

David Novella Rodriguez, Basilio del Muro-Cuellar, Olivier Sename, Martin Velasco-Villa

\section{- To cite this version:}

David Novella Rodriguez, Basilio del Muro-Cuellar, Olivier Sename, Martin Velasco-Villa. On the stabilization of high order systems with two unstable poles plus time delay. MED 2012 - 20th Mediterranean Conference on Control and Automation, Jul 2012, Barcelone, Spain. pp.12-17, 10.1109/MED.2012.6265607 . hal-00828093

\section{HAL Id: hal-00828093 https://hal.science/hal-00828093}

Submitted on 6 Jun 2013

HAL is a multi-disciplinary open access archive for the deposit and dissemination of scientific research documents, whether they are published or not. The documents may come from teaching and research institutions in France or abroad, or from public or private research centers.
L'archive ouverte pluridisciplinaire HAL, est destinée au dépôt et à la diffusion de documents scientifiques de niveau recherche, publiés ou non, émanant des établissements d'enseignement et de recherche français ou étrangers, des laboratoires publics ou privés. 


\title{
On The Stabilization of High Order Systems with Two Unstable Poles Plus Time Delay.*
}

\author{
David Novella Rodríguez ${ }^{\dagger}$, Basilio Del Muro Cuéllar ${ }^{\dagger}$, Olivier Sename $^{\ddagger}$ \\ and Martin Velasco Villa $\S$ \\ † Escuela Superior de Ingeniería Mecánica y Eléctrica, Unidad Culhuacan, \\ Instituto Politécnico Nacional, Santa Ana 1000, México D.F., 04430, México. \\ (e-mail: dnovellar@gmail.com, bdelmuro@yahoo.com). \\ ${ }^{\ddagger}$ GIPSA-Lab Department of Control Systems, Grenoble INP, ENSE3, \\ BP46 38402, Saint Martin d'Hères Cedex, GRENOBLE, FRANCE. \\ (e-mail: olivier.sename@gipsa-lab.grenoble-inp.fr). \\ $\S$ CINVESTAV-IPN, Departamento de Ingeniería Eléctrica, Sección de Mecatrónica, \\ A.P. 14-740, 07000, México D.F., México, velasco@ cinvestav.mx.
}

\begin{abstract}
This work considers the stabilization of linear time invariant high order systems with two unstable poles plus time delay. For this, we will propose a simple observer based controller in order to stabilize the system. Numerical examples and an electronic implementation of the proposed scheme are presented in order to illustrate the performance of the closed loop system.
\end{abstract}

\section{INTRODUCTION}

Systems with delays are very common and are due to several mechanisms like material or energy transport, recycling loops, etc. In addition, actuators, sensors and field networks that are involved in feedback loops usually introduce such delays, [14], [19]. Also delays can be used in model reduction where high-order (finite-dimensional) systems are approximated (in some norm sense) by low order systems with delays, [17]. It is known that timedelay is often a source of complex behaviors (oscillations, instability, bad performance), in many dynamic systems, and thus considerable attention has been paid on the stability analysis and controller design of time delay systems. Hence, there exists a great motivation to study delay effects on dynamical systems properties for two main reasons: first to understand how the delay presence may deteriorate the behavior of the system, and second to control their effects for better performance achievement on closed-loop systems, [2], [10].

Several control strategies have been developed to deal with delayed systems. A common approach is to approximate the time-delay operator by means of a Taylor or Pade series which could lead to a non minimum-phase system with rational transfer function representation. The ProportionalIntegral (PI) and Proportional-Integral-Derivative (PID) controllers are included in the control design for time delay systems [16]. On the other hand, some recent works have been devoted to the analysis of stability and stabilization

\footnotetext{
*This work was supported in part by CONACyT-México, Under Grant
} 61713 of systems with delay based on approaches of LyapunovKrasovskii and Lyapunov-Razumikhin. These results are expressed in terms of algebraic Riccati equations, [3], [19], etc., or linear matrix inequalities, [1], [4], etc.

A different approach to deal with dead time systems is the classical Smith Predictor (SP), which consists in counteracting the time delay effects by means of strategies intended to estimate the effects of current inputs over future outputs, [13], [18]. The main limitation of the original SP is the fact that the prediction scheme has not a stabilization step, which restricts its application to open-loop stable plants.

However, open-loop unstable processes arise frequently in different dynamic systems and are fundamentally difficult to control. To overcome this problem, some modifications of the SP original structure have been proposed to deal with non-stable delayed process, for instance, [15] has presented an efficient modification to the Smith predictor in order to control unstable first order system plus time delay. With a different perspective, [12] proposes a modification to the original Smith structure in order to deal with unstable first order delayed systems. Using a similar structure, the result is extended to delayed high order systems [11]. In both works, a robustness analysis is done concluding that for unstable dead time dominant systems, the closed-loop system can be unstabilized with an infinitesimal value of the modeling error, i.e., that robustness is strongly dependent on the relationship $\tau / \tau_{\text {un }}$, where $\tau$ is the process time delay and $\tau_{u n}$ the dominant unstable time-constant. For the control scheme proposed in the later works, it can be easily proven that in the case of unstable plants, the internal stability is not guaranteed. In fact it is obtained an unstable estimation error and, as a result, a minimal initial condition difference between the original plant and the model produces an internal unbounded signal.

A different approach for the stabilization problem for time delay systems has been introduced in different works, [6], [7], for instance. In these works it is proposed a solution based on the parametrization of the controllers and predictors 
of the rational part of the plant. However, the potential problem of this approach might be the implementation issue, since the resulting controllers involve FIR blocks, which are built upon Hamiltonian matrices and require matrix exponentials to be computed.

This paper is concerned with the stabilization problem of systems with two unstable poles and $m$ stable poles plus time delay. The control scheme relies on an observer-based structure, on the contrary of modified Smith predictors, the scheme only contains discrete time delay (and not distributed ones) which makes easy its practical implementation (see [19] for details on numerical implementation of modified Smith predictor scheme). This paper is organized as follows, Section 2 is dedicated to the problem formulation. Section 3 yields the preliminaries results used to obtain the main result of this work. An observer based controller is proposed in Section 4 in order to stabilize the unstable delayed system previously described, also the stability conditions of the proposed control structure are stated. Numerical simulations are presented in Section 5 to show the controller performance and the control strategy is implemented on a real electronic plant built with operational amplifiers. Finally conclusions and future perspectives are drawn.

\section{Problem Formulation}

Consider the following class of single-input single-output (SISO) linear systems with input delay:

$$
\frac{Y(s)}{U(s)}=\frac{N(s)}{D(s)} e^{-\tau s}=G(s) e^{-\tau s},
$$

where $U(s)$ and $Y(s)$ are the input and output signals respectively, $\tau \geq 0$ is the constant time delay, $N(s)$ and $D(s)$ are polynomials in the complex variable $s$ and $G(s)$ is the delay-free transfer function. Notice that with respect to the class of systems (1) a traditional control strategy based on an output feedback of the form

$$
U(s)=C(s)[R(s)-Y(s)],
$$

yields a closed-loop system given by:

$$
\frac{Y(s)}{R(s)}=\frac{C(s) G(s) e^{-\tau s}}{1+C(s) G(s) e^{-\tau s}}
$$

where the exponential term $e^{-\tau s}$ located at the denominator of the transfer function (3) leads to a system with an infinite number of poles and where the closed-loop stability properties must be carefully stated. From the classical structure of the Smith predictor, it is known that the transfer function of the closed-loop system is obtained as follows:

$$
\frac{Y(s)}{R(s)}=\frac{C(s) G(s)}{1+C(s) G(s)} e^{-\tau s},
$$

where the delay term is shifted outside of the characteristic equation of the system. Under ideal conditions, i.e., exact knowledge of the plant parameters and delay value, the SP provides a successful future estimation $\tau$ time units ahead of the $y(t)$ signal, which could be used like a control signal in a specific feedback scheme [13], [17]. Unfortunately, the classical structure of the SP is restricted to stable process. Different authors have proposed several modifications to the original SP structure to give solution to some particular cases, [11], [12], [15], [19].

This work proposes an observer based control scheme in order to stabilize a system characterized by the following transfer function:

$$
\frac{Y(s)}{U(s)}=\frac{\alpha}{(s-a)(s-b)\left(s+c_{1}\right)\left(s+c_{2}\right) \ldots\left(s+c_{m}\right)} e^{-\tau s} .
$$

Where $\tau, \quad a \quad b \quad c_{1}, c_{2} \ldots c_{m}>0$ and without loss of generality, $a \geq b>0$. The proposed control scheme has been design based on a traditional observer theory, hence, only the plant model and two static gains are enough to get an adequate estimation of an internal delay free variable which will be used in the final stabilizing control scheme.

\section{PRELIMINARY RESUlts}

Preliminary results are presented, which will be used later in order to state the stability conditions of the proposed strategy in this work.

Consider the following unstable first order system plus time delay:

$$
\frac{Y(s)}{U(s)}=G(s) e^{-\tau s}=\frac{\alpha}{s-\sigma} e^{-\tau s},
$$

with $\sigma>0$, and a proportional output feedback control as follows:

$$
U(s)=R(s)-k Y(s),
$$

which produces a closed-loop system:

$$
\frac{Y(s)}{R(s)}=\frac{\alpha e^{-\tau s}}{s-\sigma+k \alpha e^{-\tau s}} .
$$

The following result has been widely studied in the literature and the proof can be easily obtained by considering different approaches as a classical frequency domain. An alternative simple proof based on a discrete time approach is shown in [9], [8].

Lemma 1: Consider the delayed system (6) and the proportional output feedback (7). Then, there exists a proportional gain $k$ such that the closed loop system (8), is stable if and only if $\tau<\frac{1}{\sigma}$.

Now consider the high-order unstable system characterized by:

$$
G(s)=\frac{\alpha}{(s-\sigma)\left(s+\phi_{1}\right)\left(s+\phi_{2}\right) \ldots\left(s+\phi_{n}\right)} e^{-\tau s} .
$$

with $\sigma, \phi_{1}, \phi_{2} \ldots \phi_{n}>0$. With the proportional output feedback (7), the closed-loop system is as follows:

$$
\frac{Y(s)}{R(s)}=\frac{\alpha e^{-\tau s}}{\left[(s-\sigma)\left(s+\phi_{1}\right)\left(s+\phi_{2}\right) \ldots\left(s+\phi_{n}\right)\right]+k \alpha e^{-\tau s}} .
$$


Lemma 2: Consider the delayed system (9) and the proportional output feedback (7). Then, there exists a proportional gain $k$ such that the closed loop system (10), is stable if and only if $\tau<\frac{1}{\sigma}-\sum_{i=1}^{n} \frac{1}{\phi_{i}}$.

The proof of this result is presented in the Appendix A.

\section{Proposed Control Strategy}

Consider the class of systems studied in this work and characterized by the transfer function (5) with $a, b, c_{1}, c_{2}, \cdots, c_{m}>0$ and the time delay $\tau>0$, and assuming without loss of generality $a \geq b$. An observer based controller is designed in order to obtain an estimation of the internal states of the system to be used as control signals for the original process.

As a first step, the stability conditions for the controller and the observer systems are stated separately. This conditions will be used later in order to state the closed loop stability conditions for the proposed observer based controller.

\section{A. Controller Scheme}

First, taking into account the state feedback control strategy shown in the Figure 1, let us introduce the following result.

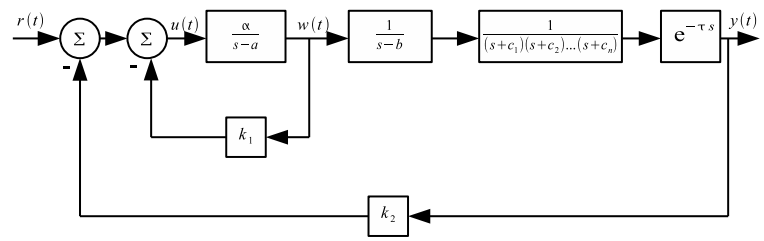

Fig. 1. Control Scheme.

Lemma 3: Consider the delayed system (5), and the state feedback controller proposed in Figure 1 . There exist constant gains $k_{1}$ and $k_{2}$ such that the closed-loop system is stable if and only if

$$
\tau<\frac{1}{b}-\sum_{i=1}^{m} \frac{1}{c_{i}}
$$

The aim of the foregoing proof is to apply the stability conditions given in Lemma 2 to the closed loop system shown in the Figure 1.

\section{Proof:}

Sufficiency. Let us consider $\tau<\frac{1}{b}-\sum_{i=1}^{m} \frac{1}{c_{i}}$. Then, $\tau=$ $\frac{1}{b}-\sum_{i=1}^{m} \frac{1}{c_{i}}-\beta$, for some $\beta>0$. Therefore, there exists $k_{1}$ such that $\beta>\frac{1}{\alpha k_{1}-a}>0$. Then $\tau<\frac{1}{b}-\sum_{i=1}^{m} \frac{1}{c_{i}}-\frac{1}{\alpha k_{1}-a}$. Finally we can conclude from Lemma 2 , where $\sigma=b, \phi_{i}=$ $c_{i}$ and $\phi_{m+1}=\alpha k_{1}-a$ there exists $k_{2}$ such that the closed loop system shown in Figure 1 is stable.

Necessity. Consider the delayed system (5), and the state feedback controller shown in Figure 1, with constant gains $k_{1}$ and $k_{2}$ such that the closed-loop system is stable. The closed loop transfer function can be written as follows:

$\frac{Y(s)}{R(s)}=\frac{\alpha e^{-\tau s}}{(s-b)\left(s+c_{1}\right) \cdots\left(s+c_{m}\right)\left(s+\phi_{m+1}\right)+\alpha k_{2} e^{-\tau s}}$,

with $\phi_{m+1}=\alpha k_{1}-a$. It is well known that a $k_{2}$ that stabilizes the delayed system (11) must also stabilize the delay free system (see for instance [5] or [10]), which implies that $\phi_{m+1}>0$. Indeed, from Lemma 2, $\tau<\frac{1}{\sigma}-\sum_{i=1}^{n} \frac{1}{\phi_{i}}$ with $\sigma=b$ and $\phi_{i}=c_{i}$, where $n=m+1$ (note that $\phi_{m+1}>0$ is a free parameter function of $k_{1}$ ). Let us consider $\beta>\frac{1}{\phi_{m+1}}>0$, denoting $\beta=\frac{1}{\sigma}-\sum_{i=1}^{m} \frac{1}{\phi_{i}}-\tau$, therefore:

$$
\tau=\frac{1}{\sigma}-\sum_{i=1}^{m} \frac{1}{\phi_{i}}-\beta<\frac{1}{\sigma}-\sum_{i=1}^{m} \frac{1}{\phi_{i}}=\frac{1}{b}-\sum_{i=1}^{m} \frac{1}{c_{i}}
$$

Note that a root locus and/or frequency domain analysis can be used to compute proper constant gains $k_{1}$ and $k_{2}$ in order to stabilize the proportional state feedback scheme.

\section{B. Observer Scheme}

In most of the practical applications, the internal variables are not measured. Thus, an observer based on an output injection strategy is proposed, as represented in Figure 2 The stability of the observer can be tackled as follows.

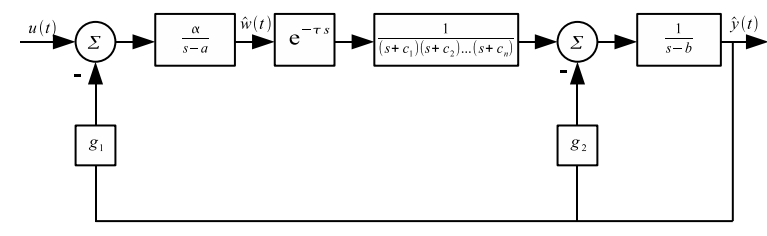

Fig. 2. Observer Scheme.

Lemma 4: Consider the delayed system (5), and the static output injection scheme shown in Figure 2 There exist constants $g_{1}$ and $g_{2}$ such that the closed-loop system is stable if and only if

Proof:

$$
\tau<\frac{1}{a}-\sum_{i=1}^{m} \frac{1}{c_{i}} .
$$

The proof can be easily derived from a dual procedure of the previous result.

As in the controller design, the computation of the proportional gains $g_{1}$ and $g_{2}$ can be solved by means of a root locus and/or a frequency domain analysis.

\section{Observer-Based Controller}

Finally, the main result of this work is presented, we propose an observed based controller as in the Figure 3 , where the observer allows to estimate the state variables, to 
be used in state feedback controller. The authors would like to stress that, in the proposed scheme, only four proportional gains are enough to get a stable closed loop behavior. As a consequence of the previous results, the following lemma can be stated.

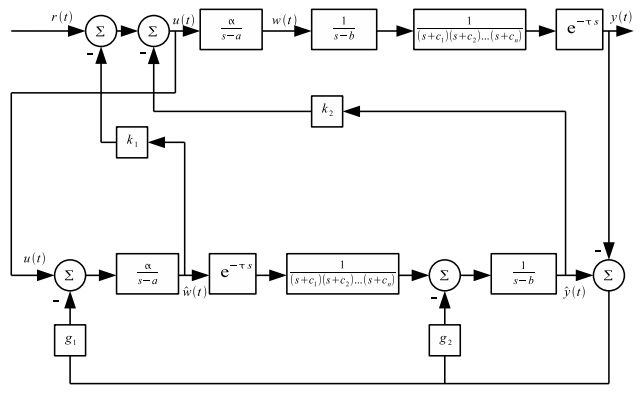

Fig. 3. Control Strategy Proposed.

Lemma 5: Consider the observer based controller scheme shown in Figure 3. There exist proportional gains $k_{1}, k_{2}, g_{1}$ and $g_{2}$ such that the closed-loop system is stable if and only if

$$
\tau<\frac{1}{a}-\sum_{i=1}^{m} \frac{1}{c_{i}}
$$

Proof: Consider the state space representation of the system (5) characterized by the following equation:

$$
\begin{gathered}
\dot{x}(t)=A_{0} x(t)+A_{1} x(t-\tau)+B u(t) \\
y(t)=C x(t)
\end{gathered}
$$

. With $x(t)=\left[\begin{array}{llllll}w(t) & x_{1}(t) & x_{2}(t) & \cdots & x_{m}(t) & z(t)\end{array}\right]^{T}$. Where,

$$
\begin{aligned}
A_{0}= & {\left[\begin{array}{ccccc}
a & 0 & \cdots & 0 & 0 \\
1 & -c_{1} & \cdots & 0 & 0 \\
\vdots & \vdots & \ddots & \vdots & \vdots \\
0 & 0 & \cdots & -c_{m} & 0 \\
0 & 0 & \cdots & 0 & b
\end{array}\right] } \\
A_{1}= & {\left[\begin{array}{ccccc}
0 & 0 & \cdots & 0 & 0 \\
0 & 0 & \cdots & 0 & 0 \\
\vdots & \vdots & \ddots & \vdots & \vdots \\
0 & 0 & \cdots & 0 & 0 \\
0 & 0 & \cdots & 1 & 0
\end{array}\right] } \\
B= & {\left[\begin{array}{c}
\alpha \\
0 \\
\vdots \\
0
\end{array}\right] } \\
C & {\left[\begin{array}{llll}
0 & &
\end{array}\right] }
\end{aligned}
$$

Note that the state state representation characterized by (12) can be returned to its transfer function representation by mean of:

$$
\frac{Y(s)}{U(s)}=C\left(s I-\left(A_{0}+A_{1} e^{-\tau s}\right)\right)^{-1} B .
$$

Which brings us back to the delayed transfer function (5). The dynamics of the estimated states and the control law can be described as follows.

$$
\begin{gathered}
\dot{\hat{x}}(t)=A_{0} \hat{x}(t)+A_{1} \hat{x}(t-\tau)+B u(t)-G(C \hat{x}(t)-y(t)) \\
u(t)=-K \hat{x}(t) .
\end{gathered}
$$

Where $\hat{x}(t)$ is the estimated state of $x(t)$, and the gain vectors $K$ and $G$ are defined by:

$$
\begin{aligned}
K & =\left[\begin{array}{llllll}
k_{1} & 0 & 0 & \cdots & 0 & k_{2}
\end{array}\right] \\
G & =\left[\begin{array}{llllll}
g_{1} & 0 & 0 & \cdots & 0 & g_{2}
\end{array}\right]^{T}
\end{aligned}
$$

Let $e(t):=x(t)-\hat{x}(t)$, then we have:

$$
\dot{e}(t)=\dot{x}(t)-\dot{\hat{x}}(t)=\left(A_{0}-G C\right) e(t)+A_{1} e(t-\tau) .
$$

And the controlled system:

$$
\dot{x}(t)=A_{0} x(t)+A_{1} x(t-\tau)-B K \hat{x}(t) .
$$

Noting $x_{e}=[x(t) e(t)]^{T}$ and after a simple manipulation of variables we have the following closed loop system with the observer and the controller proposed in the Figure 3.

$$
\begin{gathered}
\dot{x_{e}}(t)=\left[\begin{array}{cc}
A_{0}-B K & B K \\
0 & A_{0}-G C
\end{array}\right] x_{e}(t)+\cdots \\
\cdots+\left[\begin{array}{cc}
A_{1} & 0 \\
0 & A_{1}
\end{array}\right] x_{e}(t-\tau) \\
y(t)=\left[\begin{array}{ll}
C & 0
\end{array}\right] x_{e}(t) .
\end{gathered}
$$

It is easy to see that the observer based controller proposed satisfies the separation principle. Hence, the stability of the observer scheme is enough to assure an adequate error convergence, i.e. there exist proportional gains $g_{1}$ and $g_{2}$ such that $\lim _{t \rightarrow \infty}[\hat{w}(t)-w(t)]=0$ if and only if

$$
\tau<\frac{1}{a}-\sum_{i=1}^{m} \frac{1}{c_{i}} .
$$

Then, considering the fact of the observer and controller can be designed separately and reminding the stability conditions stated previously in Lemmas 3 and 4 , is clear that the observer stability condition is more restrictive than the controller one, i.e.,

$$
\frac{1}{a}-\sum_{i=1}^{m} c_{i}<\frac{1}{b}-\sum_{i=1}^{m} \frac{1}{c_{i}} .
$$

Therefore, there exist $k_{1}, k_{2}, g_{1}$ and $g_{2}$ such that the closed-loop system is stable if

$$
\tau<\frac{1}{a}-\sum_{i=1}^{m} \frac{1}{c_{i}}
$$




\section{EXAMPLES}

The following numerical examples illustrate the performance obtained by means of the observed based controller proposed.

Example 1.Consider the delayed system with two unstable poles characterized with the following transfer function:

$$
\frac{Y(s)}{U(s)}=\frac{1}{(s-0.7)(s-0.4)(s+5)(s+10)} e^{-0.5 s}
$$

Let be $a=0.7, b=0.4, c_{1}=5, c_{2}=10, \alpha=1$ and $\tau=0.5$ the parameters of the system, it is clear that the stability conditions given in Lemma 5 are satisfied, therefore there exists an observer based structure with proportional gains $k_{1}, k_{2}, g_{1}$ and $g_{2}$ such that the resulting closed-loop system is stable due to:

$$
\tau=0.5<\frac{1}{a}-\sum_{i=1}^{m} \frac{1}{c_{i}}=1.128 .
$$

As stated before, the control scheme 3 holds the separation property, hence the design of the controller and the observer is independent. Therefore, to ensure the existence of a proportional gain $k_{2}$ such that the closed loop system is stable, from Lemma 3 .

$$
k_{1}>\frac{1}{\alpha\left(\frac{1}{b}-\sum_{i=1}^{m} \frac{1}{c_{i}}-\tau\right)}+\frac{a}{\alpha}=1.28,
$$

as the value $k_{1}$ can be as large as we wish, the gain chosen is $k_{1}=20.7$, (to place the pole in $s=-20$ ), from this, after a frequency domain analysis, Nyquist stability criterion for instance, we can compute the gain $k_{2}$ such that the controller scheme shown in the Figure 3 is stable, for this example, $400<k_{2}<1721$.

The procedure to compute the observer gains $g_{1}$ and $g_{2}$ is quite similar, the proportional gain $g_{2}$ must be larger than $b$ to ensure the stability of the system. From Lemma 4 we know that

$$
g_{2}>\frac{1}{\left(\frac{1}{a}-\sum_{i=1}^{m} \frac{1}{c_{i}}-\tau\right)}+b=1.99,
$$

as the value $g_{2}$ can be as large as we wish, the gain chosen is $g_{2}=20.4$, form this, after a frequency domain analysis, we can compute the gain $g_{1}$, such that the observer scheme shown in the Figure 2 is stable, for this example, $700<$ $g_{1}<1499$.

Hence, the constant gains computed for this example are $k_{1}=20.7, \quad k_{2}=1000, g_{1}=1100$ and $g_{2}=20.4$. The Figure 4 illustrate the performance of the observer based controller in numerical simulations, the output and the error are shown respectively. The continuous line indicates the output of the closed loop system with identical initial conditions between $y(t)$ and $\hat{y}(t)$. The dashed line point
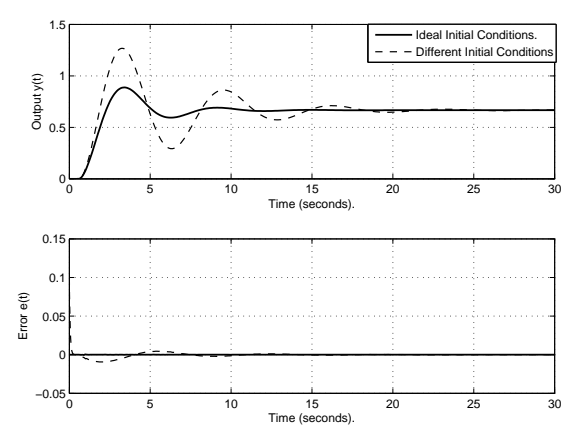

Fig. 4. Numerical Simulation Results.

to the system performance whit different initial conditions, $(\hat{y}(t)-y(t)=0.1)$.

Below, for the same example, the observed based controller is implemented by mean of the Data Acquisition System Sensoray 626, using its analog inputs/ouputs as sensors and actuators communicated with a computer through the MATLAB Real Time Workshop Toolbox. The real electronic plant is built with commercial operational amplifiers, resistors, and capacitors.

The stable output $y(t)$ of the closed loop system and the error signal $e(t)$ are shown in the Figure 5
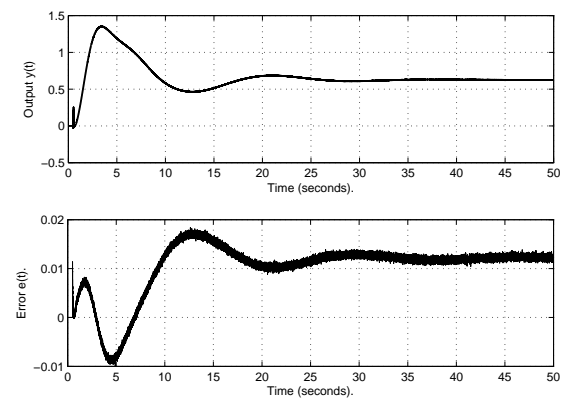

Fig. 5. Practical Implementation.

Remark 1: Notice that the controller has a efficient performance regardless the different initial conditions between the original process and the observer, and the parametrical variations due to the use of commercial electronic devices.

\section{CONClusions}

An observer based controller is proposed in this work in order to stabilize high order system with two unstable poles plus time delay. The necessary and sufficient conditions that ensure existence of the stabilizing control scheme are stated. The scheme is simple and may be easily implemented, The procedure to design the controller can be performed easily using well know analysis of linear control theory. An implementation of the observer based controller is presented by mean of an electronic plant built with operational amplifiers and the Data Acquisition system Sensoray 626. The example show the closed loop system behavior working under different initial conditions between the plant and the 
observer, as well as the parametrical variations introduced by the use of commercial electronic devices.

\section{APPENDIX}

A Proof of The Lemma 2 .

Let us consider the High-Order Unstable System with time delay given by:

$$
G(s)=\frac{\alpha}{(s-\sigma)\left(s+\phi_{1}\right)\left(s+\phi_{2}\right) \ldots\left(s+\phi_{n}\right)} e^{-\tau s} .
$$

First, analyzing the First Order Unstable Delayed System given by (6) and considering the Lemma 1 there exists a proportional gain $k$ such that a closed loop system with a simple proportional output feedback is stable if and only if $\tau<\frac{1}{\sigma}$. An analysis in the frequency domain shall confirm this result. The Nyquist stability criteria establishes that, when closing the loop whit a proportional gain $k$, the system will be stable if $0=N+P$, with $P$ being the number of poles in the right half plane " $s$ " and $N$ the numbers of clockwise round trips to the point -1 ( $N$ negative suggests round trips in the opposite direction) in the Nyquist diagram. The angle as a function of the frequency $\omega$ is given by:

$$
\angle G(j \omega)=-\left(180^{\circ}-\arctan \left(\frac{\omega}{\sigma}\right)\right)-(\omega \tau)
$$

Taking into consideration that for small frequencies $\arctan \omega \varphi \approx \omega \varphi$, it can be shown that the condition $\tau<\frac{1}{\sigma}$ is equivalent to ask that the angle path taps at least one point (for some frequency) with a value exceeding $-180^{\circ}$, that is $\angle G(j \omega)>-180^{\circ}$, i.e., one counter-clockwise round trip to the point -1 in the Nyquist diagram. Now, analyzing the system with $n=1$ characterized by:

$$
G(s)=\frac{\alpha}{(s-\sigma)(s+\phi)} e^{-\tau s}
$$

Then, there exists a proportional gain $k$ such that a closed loop system whit a simple proportional output feedback is stable if and only if $\tau<\frac{1}{\sigma}-\frac{1}{\phi}$. It can be easy to see that the Nyquist condition remains the same (one counter-clockwise round trip to the point -1 in the Nyquist diagram), now the angle condition is:

$\angle G(j \omega)=-\left(180^{\circ}-\arctan \left(\frac{\omega}{\sigma}\right)\right)-\left(\arctan \left(\frac{\omega}{\phi}\right)\right)-(\omega \tau)$

For small frequencies $\arctan \omega \varphi \approx \omega \varphi$, and starting from $\angle G(j \omega)>-180^{\circ}$ it is no difficult to conclude the relation $\tau<\frac{1}{\sigma}-\frac{1}{\phi}$. Below considering the system whit $n=2$ given by:

$$
G(s)=\frac{\alpha}{(s-\sigma)\left(s+\phi_{1}\right)\left(s+\phi_{2}\right)} e^{-\tau s} .
$$

It can be easy to see that the Nyquist condition remains the same (one counter-clockwise round trip to the point -1 in the Nyquist diagram), now the angle condition is:

$$
\begin{gathered}
\angle G(j \omega)=-\left(180^{\circ}-\arctan \left(\frac{\omega}{\sigma}\right)\right)-\left(\arctan \left(\frac{\omega}{\phi_{1}}\right)\right)-\cdots \\
\cdots-\left(\arctan \left(\frac{\omega}{\phi_{2}}\right)\right)-(\omega \tau)
\end{gathered}
$$

Again considering that for small frequencies $\arctan \omega \varphi \approx$ $\omega \varphi$, and starting from $\angle G(j \omega)>-180^{\circ}$ it is no difficult to conclude the relation $\tau<\frac{1}{\sigma}-\frac{1}{\phi_{1}}-\frac{1}{\phi_{2}}$.

This reflection can be generalized to any $n \in \mathbb{R}$ concluding that for the systems characterized by A.1 with a proportional output feedback, there exist a constant gain $k$ such that the closed loop system is stable if and only if:

$$
\tau<\frac{1}{\sigma}-\sum_{i=1}^{n} \frac{1}{\phi_{i}}
$$

\section{REFERENCES}

[1] E. Fridman and U. Shaked, An improved stabilization method for linear time-delay systems, Automatic Control, IEEE Transactions on 47 (2002), no. 11, 1931 - 1937.

[2] Keqin Gu, Vladimir L. Kharitonov, and Jie Chen, Stability of TimeDelay Systems, Birkhäuser, 2003.

[3] V.B. Kolmanovskii and J.-P. Richard, Stability of some linear systems with delays, Automatic Control, IEEE Transactions on 44 (1999), no. 5, $984-989$.

[4] Y. S. Lee, Y. S. Moon, W. H. Kwon, and P. G. Park, Delay Dependent Robust $H_{\infty}$ Control for Uncertain Systems with a State-Delay, Automatica 40 (2004), no. 1, 65-72.

[5] Efim Malakhovski and Leonid Mirkin, On Stability of Second-order Quasi-polynomials with a Single Delay, Automatica 42 (2006), 10411047

[6] L. Mirkin, On the extraction of dead-time controllers and estimators from delay-free parametrizations, Automatic Control, IEEE Transactions on 48 (2003), no. 4, 543 - 553.

[7] Leonid Mirkin and Natalya Raskin, Every stabilizing dead-time controller has an observerpredictor-based structure, Automatica 39 (2003), no. 10, 1747 - 1754

[8] J. F. Mrquez-Rubio, B. del Muro-Cullar, M. Velasco-Villa, and J. lvarez Ramrez, Stabilization strategy for unstable first order linear systems with large time-delay, Asian Journal of Control (2011), n/an/a.

[9] B. Del Muro, J.F. Márquez, M. Velasco, and J. Alvarez, Stabization strategy for unstable first order linear systems with large time delay, International Conference on Modelling Identification and Control, ICMIC (2008)

[10] Silviu-Iulian Niculescu, Delay Effects on Stability: A Robust Control Approach, Springer, 2001.

[11] J. E. Normey-Rico and E. F. Camacho, Unified approach for robust dead-time compesator design, Journal of Process Control 19 (2009), 38-47.

[12] Julio E. Normey-Rico and Eduardo. F. Camacho, Dead-time compensators: A survey, Control Engineering Practice 16 (2008), no. 4, 407428.

[13] Z. J. Palmor, Time delay compensation smith predictor and its modifications, The control Handbook (1996), 224- 237.

[14] Jean-Pierre Richard, Time-Delay Systems:An Overview of Some Recent Advances and Open Problems., Automatica 39 (2003), 1667-1694.

[15] R. A. Seshagiri, V. S. R. Rao, and M. Chidambaram, Simple analytical design of modified smith predictor with improved performance for unstable first-order plus time delay (fodtp) processes, Ind. Eng. Chem. Res 46(13) (2007), 4561-4571.

[16] Guillermo J. Silva, Aniruddha Datta, and S.P. Bhattacharyya., PID Controllers for Time-Delay Systems, Control Engineering, Birkhäuser, 2004.

[17] S. Skogestad, Simple Analytic Rules for Model Reduction and PID Controller Tunning, Journal of Process Control 13 (2003), 4.

[18] O. J. M. Smith, Close Control of Loops with Dead Time, Chem. Eng. Prog. 53 (1957), 217-219.

[19] Qing-Chang Zhong, Robust Control of Time-Delay Systems, Springer 2006. 